\title{
Project Management Using Modern Guidance, Navigation and Control Theory
}

Terry Hill - July 2010

\begin{abstract}
The idea of control theory and its application to project management is not new, however literature on the topic and real-world applications is not as readily available and comprehensive in how all the principals of Guidance, Navigation and Control (GN\&C) apply. This paper will address how the fundamental principals of modern GN\&C Theory have been applied to NASA's Constellation Space Suit project and the results in the ability to manage the project within cost, schedule and budget.
\end{abstract}

As with physical systems, projects can be modeled and managed with the same guiding principles of GN\&C as if it were a complex vehicle, system or software with time-varying processes, at times non-linear responses, multiple data inputs of varying accuracy and a range of operating points. With such systems the classic approach could be applied to small and well-defined projects; however with larger, multi-year projects involving multiple organizational structures, external influences and a multitude of diverse resources, then modern control theory is required to model and control the project.

The fundamental principals of GN\&C stated that a system is comprised of these basic core concepts: State, Behavior, Control system, Navigation system, Guidance and Planning Logic, Feedback systems. The state of a system is a definition of the aspects of the dynamics of the system that can change, such as position, velocity, acceleration, coordinate-based attitude, temperature, etc. The behavior of the system is more of what changes are possible rather than what can change, which is captured in the state of the system. The behavior of a system is captured in the system modeling and if properly done, will aid in accurate system performance prediction in the future. The Control system understands the state and behavior of the system and feedback systems to adjust the control inputs into the system. The Navigation system takes the multiple data inputs and based upon a priori knowledge of the input, will develop a statisticalbased weighting of the input to determine where the system currently is located. Guidance and Planning logic of the system with the understanding of where it is (provided by the navigation system) will in turn determine where it needs to be and how to get there. Lastly, the system Feedback system is the right arm of the control system to allow it to affect change in the overall system and therefore it is critical to not only correctly identify the system feedback inputs but also the system response to the feedback inputs. And with any systems project it is critical that the objective of the system be clearly defined for not only planning but to be used to measure performance and to aid in the guidance of the system or project. 
The same system principals discussed above can and have been applied to current NASA projects and the principles can be mapped to real-world constituents allowing project managers to apply systems theories well defined in engineering and mathematics to a discipline (project management) that historically has been based in personal experience and intuition. With this mapping of GN\&C theory to project management, it will allow a direct and methodical approach to project management and control resulting in predicable project performance and understanding of the project constraints, how it can be controlled and the impacts to external influences and inputs. To a project manager this flows down to the three bottom-line variables: cost, schedule, scope and what combination of the three need to be modified to reach a successful project performance and completion. 